\title{
LA SIGNIFICATION DE L'ARTICLE DANS LES NOMS DE LIEUX ${ }^{1}$
}

\author{
Par Maurice-Ed. Perret
}

En Suisse romande, de même qu'en France et dans tous les pays de langue française, un certain nombre de noms de lieux habités sont précédés d'un article défini; nous avons ainsi, en Suisse: le Brassus, la Neuveville, le Locle; en France: le Havre, la Rochelle, le Creusot; en Belgique: la Louvière, la Roche; au Canada: la Tuque, l'Epiphanie $^{2}$. Il semble à première vue que ce n'est qu'un effet du hasard, mais si nous examinons la répartition géographique des noms avec article en Suisse romande, nous constatons, à quelques exceptions près, qu'ils sont groupés.

Prenons tout d'abord les noms de communes avec article. Dans le Valais, il n'y en a qu'un: les Agettes, village situé à 1200 mètres d'altitude. Dans le canton de Genève, on n'en trouve aucun. Dans le canton de Vaud, une ou deux communes au bord du Léman ont un article: la Tour-de-Peilz, le Châtelard entre autres; de même qu'une ou deux communes dans le Gros de Vaud, en particulier la Sarraz; par contre, les noms avec article sont plus nombreux dans la région du Jorat et du Mont-Pélerin - le Mont, les Thioleyres, les Tavernes, les Cullayes - et surtout au pied du Jura - la Rippe, le Muids, le Vaud, la Praz, les Clées, l'Abergement - et dans le Jura même: l'Abbaye, le Lieu, le Chenit. Dans le canton de Fribourg, il y a quelques communes isolées et des communes autour des massifs du Moléson, du Gibloux et de la Berra: le Crêt, la Joux, le Châtelard, le Pâquier, la Tour-de-Trême, la Roche. Dans le canton de Neuchâtel, toutes les communes des montagnes, par exemple la Côte-aux-Fées, les Bayards, le Cerneux-Péquignot, la Chaux-de-Fonds, et les trois communes les plus élevées du Val-de-Ruz: les Hauts-Geneveys, les Geneveys-sur-Coffrane, le Pâquier. Dans le Jura bernois, presque toutes les localités des Franches-Montagnes, en outre la Heutte.

Parmi les noms des hameaux et des fermes isolées, nous remarquons que l'article est de règle dans les communes qui ont elles-mêmes un nom avec article. Il y en a, en plus, un assez grand nombre dans le Gros de Vaud et le canton de Fribourg, mais presque aucun dans le canton de Genève, dans le canton du Valais, sur les bords du Léman et en Ajoie. En examinant les régions où se trouvent concentrés les noms avec article, nous trouvons facilement l'explication de ce fait: ce sont les massifs montagneux, les vallées écartées, par conséquent des régions défrichées et colonisées plus tardivement que le reste du pays. L'on en peut donc conclure que les localités, les domaines, les fermes avec un nom précédé de l'article sont d'origine relativement récente; il est du reste possible de le vérifier pour toutes les localités dont la date de fondation est connue. On remarque aussi que les localités avec article, qui sont d'origine rurale, sont toutes dans des situations moins favorables au point de vue de l'agriculture que les autres, soit comme nous l'avons vu, dans des massifs montagneux (le Jorat, le Gibloux, les chaînes du Jura), ou des vallées écartées (la vallée de Joux, la vallée de la Brévine, la vallée de la Chaux-de-Fonds), soit sur des plateaux élevés comme les Franches-Montagnes, soit encore au fond de vallées resserrées et peu ensoleillées (la Heutte, la Reuchenette, l'Etivaz, la Sonnaz, le Brocard) ou des régions marécageuses (la Vounaise - nom qui signifie le marais -; la Rougève et la Rogivue, localités situées de part et d'autre d'un marais). Quelques localités, en plaine, ont aussi des noms avec article; elles sont aussi d'origine assez récente; ce sont en particulier la Neuveville, fondée par l'Evêque de Bâle en 1312, le Landeron, ville fondée par le comte Rodolphe de Neuchâtel en 1325,

${ }^{1}$ Communication présentée à l'Assemblée générale de la Société helvétique des Sciences naturelles, le 5 septembre 1948, à Saint-Gall.

2 Dans d'autres langues, on rencontre l'article dans des noms de lieux, surtout en espagnol ( $\mathrm{La} \mathrm{Paz}$, Los Angeles), mais aussi en portugais (O Porto), en italien (La Spezia), en anglais (The Rock, Australie), en hollandais ('t Zand). En Suisse allemande, l'article est employé dans le langage populaire devant les noms de lieux dont le sens est encore compris ou l'était récemment, par exemple, dans le canton de Berne on dit: a der Lenk, uf der Rüti, a der Lauene, mais la forme officielle omet l'article (communication du professeur J. U. HuBsChmied). 


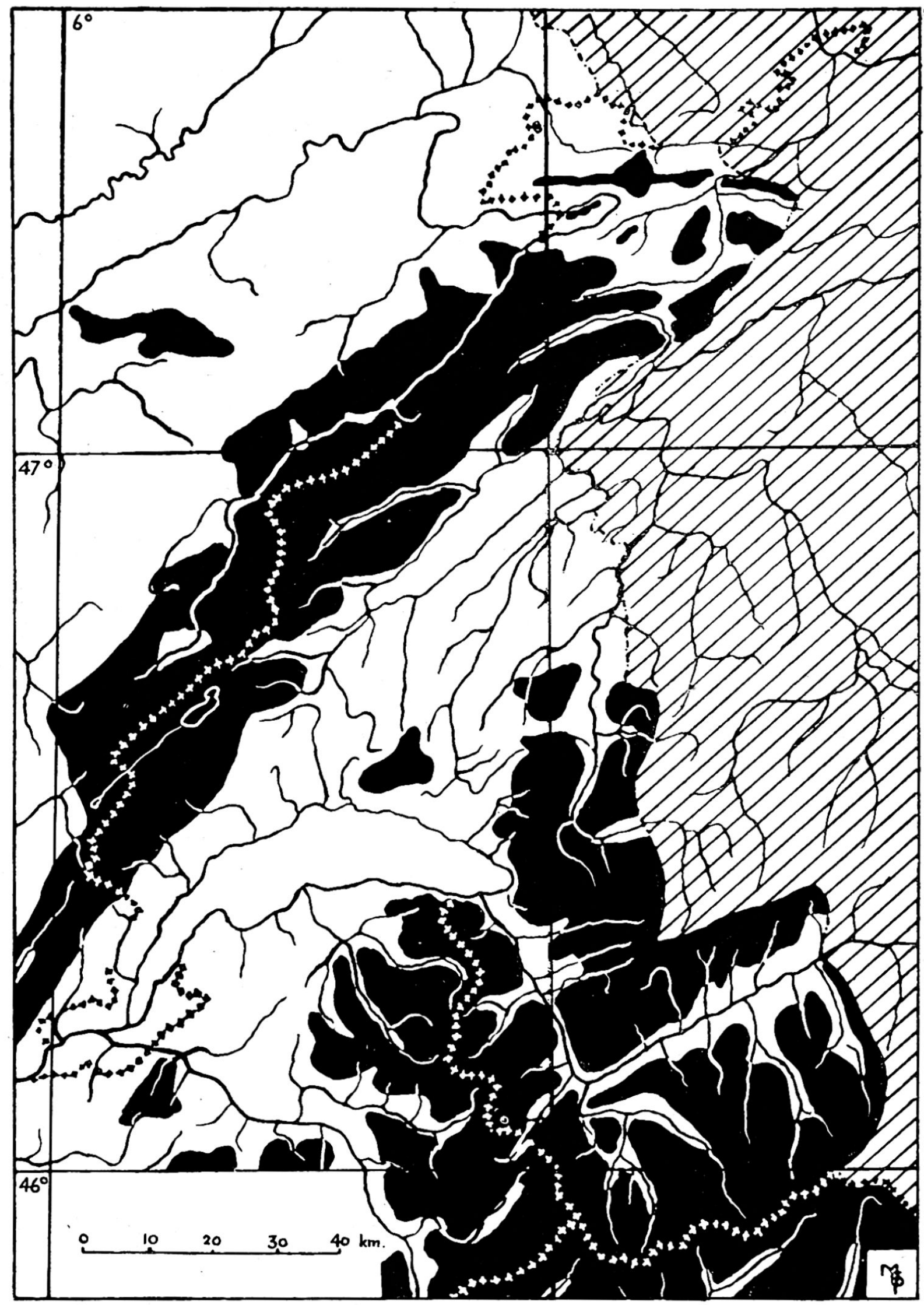

Le peuplement de la Suisse française et des régions voisines au Xe siècle. Les surfaces noires sont les régions où tous les noms de lieux habités sont précédés d'article; on en peut conclure que ces régions étaient inhabitées au Xe siècle 
les Tavernes, localité qui eut pour origine l'auberge fondée au XIVe siècle pour loger les hôtes de l'abbaye de Haut-Crêt, les Thioleyres qui étaient autrefois les tuileries déperidant de cette abbaye qui fut elle-même fondée en 1143. Quelques localités fondées tardivement tirent leur nom d'une tour fortifiée ou d'un château plus ancien autour desquels elles ont été construites; c'est probablement le cas de la Tour-de-Peilz, la Tour-de-Trême, la Sarraz, le Châtelard (Vaud et Fribourg).

L'usage de désigner une localité par un nom avec article a dû s'établir définitivement à une époque déterminée, mais en deux fois: Tout d'abord l'article n'a été donné qu'à des noms simples; on peut vérifier la chose en France. Aux XIIe et XIIIe siècles, de, nombreuses villes nouvelles ont été fondées, et l'on connaît en général la date exacte de leur fondation. C'est ainsi que l'on trouve diverses localités avec les noms de la Ferté, la Roche, la Bastide, la Bâtie, avec article, tandis qu'à la même époque l'on a divers Villeneuves, Villefranches, Montforts, Châteauneufs, sans article. L'usage de l'article s'est ensuite étendu aux noms composés, c'est ainsi que l'on a plus tard la Neuveville en 1312 - alors que Villeneuve dans le canton de Vaud datait de 1214 -, le Noirmont, le Peuchapatte. L'habitude de donner des noms sans article s'est maintenue jusqu'à nos jours dans certains cas, par exemple avec les noms de saints, et pour certaines formes comme Bellevue, Montbrillant, ou encore pour des localités qui ont reçu un nom rappelant une autre ville, par exemple Jérusalem et Gibraltar, quartiers de la Chaux-deFonds; encore aujourd'hui, un propriétaire ou un hôtelier nommera instinctivement sa maison Beau-Rivage, Jolimont, Roche Fleurie, Hôtel Beau-Séjour, sans article puisque ce sont des noms composés, mais Les Lilas, L’Ermitage, Hôtel du Lac, Hôtel des Glaciers, puisqu'il s'agit de noms simples.

Comme toute règle, celle de l'article présente des exceptions, des cas spéciaux: certaines localités ont changé de nom à une époque ou une autre, ainsi l'Isle s'appelait autrefois Chablie, la Robellaz (Vaud) Villar Luchon et le Villaret (Vaud) Villar Frelon. Dans quelques cas, l'article s'est soudé au nom: les Soz devenu Lessoc, la Joux devenu Lajoux, Lourtier au lieu de l'Ourtier. On a aussi ajouté un article à un nom ancien, en particulier lorsqu'il s'est agi de distinguer deux parties d'une localité ou deux villages homonymes: le Grand-Saconnex et le Petit-Saconnex, Villars-le-Grand, Villars-le-Comte, Villars-lesMoines, Vufflens-la-Ville et Vufflens-le-Château, mais il est facile de reconnaître ces cas exceptionnels.

Quant à la date de l'introduction de l'article dans les noms de lieu, il est difficile de la fixer avec précision, du moins pour les noms simples, car les documents historiques sont peu nombreux pour cette époque. Un acte mentionne vers 968 une «firmitas» devenue plus tard la Ferté; en 1066, on trouve la mention d'un "turriculum», nom latinisé d'une localité qui s'appelle la Toureille. La date de l'introduction de l'article dans les noms composés peut, par contre, être fixée assez exactement; on trouve en effet, parmi les villes et monastères fondés entre le XIIe et le XVe siècle, les noms suivants: Bonmont en 1123, Hauterive et Humilimont en 1137, Haut-Crêt et Fontaine-André en 1143, Villeneuve en 1214, tous sans article, et plus tard la Maigrauge en 1255, la Fille-Dieu en 1268, la Valsainte en 1292, la Part-Dieu en 1307, la Neuveville en 1312 et le Landeron en 1325. C'est donc dans la première moitié du XIIIe siècle que l'usage de l'article s'est généralisé.

$\mathrm{Si}$, faisant abstraction des noms composés et des, exceptions que nous connaissons, nous délimitons sur une carte (figure ci-jointe) les régions où tous les noms simples ont un article, nous obtenons ainsi une image de l'extension du peuplement vers la fin du Xe siècle. Les documents historiques viennent confirmer notre théorie. Ils nous apprennent que, jusqu'au Xe siècle, le Jura, le Jorat, le Gibloux étaient encore des déserts. Les découvertes archéologiques servent aussi de preuve: On n'a trouvé aucun vestige de localité romaine ou burgonde dans les régions indiquées en noir sur la carte; par contre, des fouilles ont montré que les vallées des Alpes étaient peuplées dès les temps préhistoriques et que dans cette partie du pays les régions habitées ont peu changé depuis le moyen âge.

La comparaison de la carte du peuplement vers le Xe siècle et de la carte des formes de l'habitat rural en Suisse ${ }^{3}$ nous permet de faire une nouvelle constatation intéressante, c'est que toutes les zones de dispersion et la plupart des zones de transition avec villages, hameaux et fermes intercalaires, étaient iencore désertes au Xe siècle. La dispersion, en Suisse romande, est donc d'origine relativement récente.

${ }^{3}$ Cf. Carte de l'habitat rural, d'après la carte de Ch. Biermann, complétée par F. Nussbaum et P. Vosseler, dans J. Frür: Géographie de la Suisse, tome II, planche IV. Lausanne 1939. 
On voit donc que l'article est important; il fait partie intégrante des noms de lieux, où il se trouve et il ne devrait jamais être omis ou ajouté arbitrairement.

Principales sources utilisées: Dauzat, A.: La Toponymie française. Paris 1939. - LoNGnon, A.: Les noms de lieu de la France. Paris 1929. - Dellenbach, M.: La conquête du massif alpin et de ses abords par les populations préhistoriques. Grenoble 1935. - MUrET, E.: Les noms de lieu dans les.langues romanes. Paris 1930. - Chartes et documents dans les Mémoires et Documents publiés par la Société d'histoire de la Suisse romande. - Dictionnaire géographique de la Suisse. Neuchâtel 1902-1910. - Dictionnaire historique et biographique de la Suisse. Neuchâtel 1921-1934. — Schweizerisches Ortschaftenverzeichnis / Dictionnaire des localités de la Suisșe. Bem 1895. - Ortsbuch der Schweiz / Dictionnaire des localités de la Suisse. Bern 1928.

\title{
L'ARTICOLO DETERMINATO DAVANTI AI NOMI DI LOCALITA
}

Nella Svizzera tedesca e francese molti nomi di località sono preceduti dall'articolo determinato. La carta mostra che questi nomi sono distribuiti a gruppi, soprattutto nel Giura e nell'Altipiano montuoso. Queste località erano già cosi denominate nella cartografia del secolo $\mathrm{X}$ che rispecchia la situazione degli abitati del secolo precedente. Il raffronto con una carta attuale dimostra che le denominazioni assumono distribuzione sparsa soltanto in quelle regioni che prima del secolo X erano ancora improduttive, di modo che esse diventerebbero relativamente recenti. L'autore conclude che quando è presente, l'articolo determinato fa parte integrante del nome e di conseguenza non può essere aggiunto o tralasciato ad arbitrio.

\section{DIE BEDEUTUNG DES ARTIKELS VOR ORTSNAMEN}

Vielen Ortsnamen welschschweizerischer und französischer Gebiete geht ein bestimmter Artikel voran. Die Karte zeigt, daß diese Namen gruppenweise auftreten, besonders im Jura und im bergigen Mittelland. Sie bezeichnen Siedlungen, die seit dem 10. Jahrhundert begründet wurden. Die Karte entspricht somit einer Siedlungskarte des 9. Jahrhunderts. Ihr Vergleich mit einer Karte der aktuellen Siedlungsformen erweist nämlich, daß Streusiedlung nur in Landesteilen erfolgte, die vor dem 10. Jahrhundert noch öde waren und da $\beta$ sie daher relativ jung ist. - Wo der bestimmte Artikel vorkommt, ist er somit ein wesentlicher Teil der Ortsnamen und sollte nie willkürlich weggelassen oder hinzugefügt werden.

\section{ZUR ENTWICKLUNG DES SCHWEIZER FLUSSNETZES}

\author{
Von Edwin Hennig
}

Mit 3 Karten

Der landschaftliche Formenschatz der Erdoberfläche - uns oft mit seinem Frieden beglückend - ist, aufs große gesehen, eine Kampffront zwischen den von unten her andrängenden Kräften der Hebung und (bei Vulkanen) Aufschüttung und den aus der Sonnenenergie gespeisten atmosphärischen Einflüssen der Niederschläge, Luftströmungen, Temperaturschwankungen von außen her. Unterschiedliche Widerstandsfähigkeit der Gesteinsabsätze bedingt die Einzelheiten des Reliefs. Das abfließende Wasser muß sich vorhandenen Gegebenheiten anpassen, formt sie aber im einzelnen in beachtlichem Umfange mit. Als feine Libelle antworten die Gewässer in ihrem Verlaufe und ihrer Auswirkung auf zarteste Regungen der Erdkruste; ihre Veränderungen verraten uns, was in bloßem Schichtfallen gar nicht oder nicht hinreichend davon sichtbar wird.

Wie wir im Echo der Ablagerungen in der Vortiefe des Alpenzuges über die Vorgänge im Gebirgskörper mehr erfahren als aus dessen stolzen Ruinen selber, so können wir das Auf und $A b$, das Drängen und die Schiefstellungen der Vortiefe selbst am besten ablesen aus dem abenteuerlich wechselvollen Werdegange ihrer Entwässerung. Als Wellental vor dem sich nordwärts wälzenden Wellenberge des Alpenkörpers wurde auch die randliche Senke gleichermaßen abgedrängt, ihre frühen Absätze in den Hebungsakt hineinbezogen, neue Regionen ihr einverleibt, der Raum als Ganzes mehrfach angehoben, um wieder zurückzusinken, und in sich schief gestellt und gefältelt. Dem haben sich die Wasserläufe , anzupassen; sie wurden vor immer neue Bedingungen gestellt.

In frühesten Phasen hatte die Alpenfaltung noch am Meeresboden begonnen, führte zu Schwellen, zu Inselzügen, vollzog sich aus einem mehr und mehr verengten Senkungstroge. Als sich der auftauchende Archipel langsam zu einheitlicherer Landmasse herauswölbte, die Haupthebung dem Fal- 